\title{
Persistent high BAL fluid granulocyte activation marker levels as early indicators of bronchiolitis obliterans after lung transplant
}

\author{
G.C. Riise*, B.A. Andersson**, C. Kjellström ${ }^{+}$, G. Martensson*, F.N. Nilsson ${ }^{++}$, \\ W. Ryd ${ }^{\dagger}, H$. Scherstén ${ }^{++}$
}

Persistent high BAL fluid granulocyte activation marker levels as early indicators of bronchiolitis obliterans after lung transplant. G.C. Riise, B.A. Andersson, C. Kjellström, G. Martensson, F.N. Nilsson, W. Ryd, H. Scherstén. (C) ERS Journals Ltd 1999.

ABSTRACT: The major cause of mortality in the long-term in lung transplant recipients is chronic rejection. This is a fibroproliferative process in the small airways leading to obliterative bronchiolitis and progressive loss of lung function, both constituting the clinical entity bronchiolitis obliterans syndrome (BOS). Granulocyte activation has been implicated as one factor behind BOS. Granulocyte markers in bronchoalveolar lavage (BAL) fluid were prospectively and longitudinally studied in order to identify possible association with BOS.

BAL fluid from 266 bronchoscopy procedures performed in twelve single lung, eight bilateral lung and five heart/lung transplant recipients were analysed. The majority (19 of 25) were studied for a period of 2 yrs after surgery. Myeloperoxidase (MPO), eosinophil cationic protein (ECP) and interleukin-8 (IL-8) levels were used as indirect markers of activation and attraction of granulocytes.

Five patients developed BOS. Ninety-eight episodes of acute rejection, nine of bacterial infection, 19 of cytomegalovirus pneumonitis, nine of Pneumocystis carinii infection, two of aspergillus infection and two of respiratory syncytial virus infection were diagnosed. BOS patients had significantly higher mean levels of MPO, ECP and IL-8 compared to patients without BOS, irrespective of acute rejection status. Over time, the five patients with BOS had significantly elevated BAL fluid levels of MPO and ECP as well as neutrophil percentages, and in four patients this increase preceded the clinical diagnosis of BOS by several months.

Elevated bronchoalveolar lavage fluid neutrophil percentage as well as levels of the granulocyte activation markers myeloperoxidase and eosinophil cationic protein appear to be early signs of development of $\mathrm{BOS}$ in lung transplant recipients. Eur Respir J 1999; 14: 1123-1130.
*Dept of Pulmonary Medicine, **Dept of Clinical Immunology, ${ }^{+}$Dept of Pathology, ${ }^{++}$Dept of Cardiothoracic Surgery, ${ }^{\dagger}$ Dept of Cytology, Sahlgrenska Univ. Hospital, University of Gothenburg, Sweden.

Correspondence: G.C. Riise

Dept of Pulmonary Medicine

Sahlgrenska University Hospital

S-413 45 Göteborg

Sweden.

Fax: 4631824904

Keywords: Lung transplantation myeloperoxidase

neutrophils

obliterative bronchiolitis rejection

Received: September 101998

Accepted after revision July 161999

This study was supported by the Swedish Heart and Lung Foundation and the Medical Society of Gothenburg, Sweden.
Lung transplantation has become an accepted therapy in end-stage lung disease, but despite improvements in organ preservation, surgical technique, immunosuppressive treatment and infection control, 5-yr survival is still $<50 \%$ [1].

The major cause of mortality in the long-term is the advent of chronic rejection, which can affect up to $50 \%$ of patients surviving $>3$ months after transplantation [2]. This is a fibroproliferative process in the small airways leading to the characteristic histopathological changes of obliterative bronchiolitis (OB) in lung biopsy samples and a progressive refractory loss of lung function [3]. The major risk factors for developing $\mathrm{OB}$ are frequent acute rejections during the early postoperative period [4], cytomegalovirus (CMV) infection and a preoperative diagnosis of primary pulmonary hypertension [5]. Since OB is easily overlooked or not even present in transbronchial lung biopsy (TBB) samples [6], the clinical diagnosis of bronchiolitis obliterans syndrome (BOS) is based on either spirometric and/or histopathological biopsy findings [3].

Early diagnosis of BOS using pulmonary function tests or TBB is important since immediate augmented immunosuppressive treatment has led to increased survival rates [7]. However, except for successive reduction in lung function, no clinical variable has so far been identified that is able to predict and monitor development of BOS. For this purpose, in analogy with results from other fields of lung research, specific biomarkers reflecting activation of inflammatory cells in the lung have been studied in lung transplant recipients.

Recently, bronchoalveolar lavage (BAL) fluid levels of the neutrophil-activating cytokine interleukin-8 (IL-8), the neutrophil oxidative enzyme myeloperoxidase (MPO) and the eosinophil activation marker eosinophil cationic protein (ECP) were found not to be good indicators of acute allograft rejection [8]. In contrast, patients with manifest BOS proved to have significantly increased levels of both IL-8 and MPO, as well as neutrophil percentages, in BAL fluid compared to matched controls without BOS [9]. Furthermore, DiGiovine et al. [10] reported from neutrophil chemotaxis experiments that IL-8 accounted for a significant amount of the neutrophil chemotactic activity found in lung transplant recipients with BOS.

The present study was designed to prospectively and longitudinally study BAL fluid inflammatory cells as well 
as their corresponding activation markers in lung transplant recipients. The aim was to identify potential patterns of these variables associated with development of BOS, and to determine whether they could be used as early predictors of this process.

\section{Methods}

\section{Subjects}

Twenty-five consecutive patients who underwent single lung $(n=12)$, bilateral lung $(n=8)$ or heart/lung $(n=5)$ transplantation between September 1994 and April 1996 were studied prospectively. Of these, 19 patients were studied for a period of $2 \mathrm{yrs}$, five for 18 months and one 16 months after surgery. One patient underwent single-lung retransplantation early in the postoperative period. Three patients were not included in the study due to early postoperative deaths (within 1 month post-transplant). For detailed patient characterization, see table 1 .

Donors and recipients were matched for CMV serological status. All organs were harvested in a similar fashion. Surgical procedures, immunosuppression therapy and CMV prophylaxis (Ganciclovir $5 \mathrm{mg} \cdot \mathrm{kg}^{-1}$ twice daily for 2 weeks, thereafter $5 \mathrm{mg} \cdot \mathrm{kg}^{-1}$ once daily for 2 weeks) were performed as earlier described [11, 12]. Episodes of acute rejection were treated with methylprednisolone (1 g) i.v. for 3 days, and, in cases of resistance to therapy, rabbit antithymocyte globulin (ATG, Pasteur Merieux, Lyon, France) treatment was instituted.

The study design was approved by the ethical committee of University of Gothenburg, and all subjects gave their consent after both written and oral information.

\section{Postoperative follow-up}

Surveillance bronchoscopy using TBB and BAL were performed according to protocol at 2, 4, 8 and 12 weeks and at $6,9,12,18$ and 24 months after surgery. Ventilatory lung function (forced expiratory volume in one second FEV1)) was registered using a Bernstein spirometer (Vitalograph, Burkingham, UK) or a Vitalograph (Vitalograph) starting 4 weeks post-transplant and continuing at identical protocol intervals.

Additional TBB and BAL were performed $\sim 4$ weeks after augmented immunosuppressive treatment of rejection episodes, or whenever clinical parameters indicated a deterioration in patient status. Fibreoptic bronchoscopy was performed via the oral route after oropharyngeal lignocaine anaesthesia and intravenous propofol sedation. Supplemental $100 \%$ oxygen was delivered nasally at a rate of $4-5$ $\mathrm{L} \cdot \mathrm{min}^{-1}$, with blood oxygen saturation continually monitored using an Ohmeda pulse oximeter (Ohmeda, Louisville, KY, USA).

A histopathological diagnosis of rejection was based on the assessment of TBB and BAL samples. The evaluation of acute rejections followed the recommendations of the Lung Rejection Study Group of the International Society for Heart \& Lung Transplantation [13, 14], and chronic rejection with $\mathrm{OB}$ the grading system established by "a working formulation for the standardization of nomenclature and for clinical staging of chronic dysfunction in lung allografts" [3]. BOS was defined as an irreversible decline in FEV1 of $\geq 20 \%$ of baseline value, which was determined as the mean maximum FEV1 from two consecutive measurements 30 days apart during the first postoperative year. According to guidelines, histopathological changes of $\mathrm{OB}$ on $\mathrm{TBB}$ are not a requirement for diagnosis but can be added [3].

BAL fluid analysis included direct microscopy for the detection of CMV inclusion bodies, Pneumocystis carinii, fungi and mycobacteria. Immunocytochemical techniques for the identification of $P$. carinii, CMV and Legionella pneumophila in BAL fluid and/or TBB were applied routinely. Cultures for bacteria including legionella and mycobacteria, fungi and virus were performed, and the presence of the CMV and respiratory syncytial virus (RSV) genome were investigated by polymerase chain reaction amplification. Diagnosis of CMV pneumonitis was based on histopathological changes of alveolitis on TBB together with the presence of CMV inclusion bodies in TBB and/or BAL samples. Diagnosis of $P$. carinii pneumonia (PCP) was based on demonstration of the organism by means of immuno- or silver staining of TBB and BAL samples. Diagnosis of bacterial infection was based on the presence of significant bacterial growth from BAL samples $\left(>10^{5}\right.$ $\left.\mathrm{CFU} \cdot \mathrm{mL}^{-1}\right)$. In addition, the presence of occult bacterial infection was assessed by means of regular monitoring of blood C-reactive protein (CRP) and leukocyte levels, repeated chest radiography, and clinical evaluation of fever, malaise or failure to thrive.

Table 1. - Clinical characteristics of 20 transplant recipients without and five with bronchiolitis obliterans syndrome (BOS))

\begin{tabular}{|c|c|c|c|c|c|}
\hline Presurgical diagnosis & Patients $\mathrm{n}$ & Sex & Surgical procedure & Age yrs (range) & $\mathrm{BOS}^{\#}$ \\
\hline \multicolumn{6}{|l|}{ Patients without BOS* } \\
\hline Emphysematous disease & 9 & $6 \mathrm{~F} / 3 \mathrm{M}$ & Single lung & $50(43-54)$ & - \\
\hline Emphysematous disease & 1 & $1 \mathrm{~F}$ & Bilateral lung & 49 & - \\
\hline Idiopathic pulmonary fibrosis & 1 & $1 \mathrm{~F}$ & Single lung & 51 & - \\
\hline Eisenmenger's syndrome & 3 & $1 \mathrm{~F} / 2 \mathrm{M}$ & Heart/lung & $35(19-40)$ & - \\
\hline Primary pulmonary hypertension & 3 & $1 \mathrm{~F} / 2 \mathrm{M}$ & Bilateral lung & $44(25-53)$ & - \\
\hline Primary pulmonary hypertension & 1 & $1 \mathrm{M}$ & Heart/lung & 21 & - \\
\hline Cystic fibrosis & 2 & $1 \mathrm{~F} / 1 \mathrm{M}$ & Bilateral lung & $24(23-25)$ & - \\
\hline \multicolumn{6}{|l|}{ Patients with $\mathrm{BOS}^{+}$} \\
\hline Emphysematous disease & 1 & $1 \mathrm{~F}$ & Single lung & 46 & $2 b$ \\
\hline Emphysematous disease & 1 & $1 \mathrm{~F}$ & Bilateral lung & 51 & $3 b$ \\
\hline Idiopathic pulmonary fibrosis & 1 & $1 \mathrm{~F}$ & Single lung & 49 & $2 b$ \\
\hline Primary pulmonary hypertension & 1 & $1 \mathrm{~F}$ & Bilateral lung & 25 & $3 b$ \\
\hline Primary pulmonary hypertension & 1 & $1 \mathrm{~F}$ & Heart/lung & 44 & $3 b$ \\
\hline
\end{tabular}

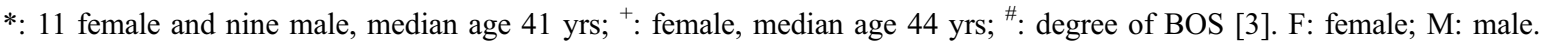




\section{Collection of samples}

All bronchoscopy was performed between $8: 30 \mathrm{~h}$ and 10:30 h. BAL was performed by infusion of eight $20-\mathrm{mL}$ warmed sterile pyrogen-free phosphate-buffered saline (PBS) aliquots into a segmental middle lobe or lingula bronchus with the bronchoscope in a wedged position. The fluid was aspirated after each $80 \mathrm{~mL}$ of infusion, pooled in a sterile siliconized container and immediately transported on ice to the laboratory. Cellular components were sedimented via centrifugation for $10 \mathrm{~min}$ at $500 \times g$ at $4{ }^{\circ} \mathrm{C}$, and the supernatant removed and frozen at $-70^{\circ} \mathrm{C}$. Before subsequent analysis of inflammatory markers, an additional 10 min centrifugation at $10,000 \times g$ was performed.

Cytocentrifuge slides (Shandon Southern Products, Runcorn, UK) were prepared using $100-\mu \mathrm{L}$ aliquots of the resuspended cell pellet. Slides were immediately fixed in $96 \%$ alcohol and stained with May-Grünwald Giemsa for later identification of cell types on a morphological basis. Percentages of polymorphonuclear granulocytes, eosinophil granulocytes, macrophages and lymphocytes were calculated after counting 200 cells using a standard light microscope. All samples were analysed by the same trained cytology assistant in a blinded manner.

TBBs were routinely taken after BAL. At least five macroscopically adequate biopsy specimens were sampled under fluoroscopic guidance from different sites within one lung using alligator forceps, immediately placed in $10 \%$ buffered formalin and sent for histopathological analysis.

\section{Analysis of inflammatory markers}

Radioimmunoassay of MPO, ECP and albumin (Pharmacia \& Upjohn Diagnostics, Uppsala, Sweden) levels in BAL fluid were performed according to the instructions of the manufacturers. The inter- and intracoefficients of variation of the radioimmunoassays used in the study have been investigated and allowed for in the assay instructions issued by the manufacturers. The lower limit of detection was $8 \mu \mathrm{g} \cdot \mathrm{L}^{-1}$ for MPO, $2 \mu \mathrm{g} \cdot \mathrm{L}^{-1}$ for ECP and $3 \mu \mathrm{g} \cdot \mathrm{L}^{-1}$ for albumin.

IL-8 was measured using commercial antibody pairs (ImmunoKontact, Frankfurt am Main, Germany) and recombinant IL-8 (R \& D Systems Europe, Abingdon, UK) and the following enzyme-linked immunosorbent assay (ELISA) protocol. The capture antibody, $2 \mu \mathrm{g} \cdot \mathrm{mL}^{-1}$ in 0.1 $\mathrm{M} \mathrm{NaHCO}$ buffer, pH 8.2, was used to coat a 96-well plate (Maxisorp; A/S Nunc Roskilde, Denmark) overnight at $4{ }^{\circ} \mathrm{C}$. After two washes with PBS containing $0.05 \%$ Tween 20 , the plate was blocked with $3 \%$ bovine serum albumin (BSA) in PBS for $2 \mathrm{~h}$ at room temperature. The samples were added and the plate incubated overnight at $4{ }^{\circ} \mathrm{C}$. After four washes with PBS-Tween, the biotinylated antibody, $1 \mu \mathrm{g} \cdot \mathrm{mL}^{-1}$ in PBS BSA, was added and the plate incubated for $1 \mathrm{~h}$ at room temperature $\left(20^{\circ} \mathrm{C}\right)$. After six washes, extravidin (Sigma, St. Louis, MO, USA; $4 \mu \mathrm{g} \cdot \mathrm{mL}^{-1}$ in PBS) was added and the plate incubated for $1 \mathrm{~h}$ at room temperature. After eight washes, phosphate substrate ( $p$ nitrophenylphosphate, Sigma, $1 \mathrm{mg} \cdot \mathrm{mL}^{-1}$ in $1 \mathrm{M}$ diethanolamine buffer) was added, and the plate read at $405 \mathrm{~nm}$ in a spectrophotometer (Titertek Multiscan; Flow Laboratories Irvine, UK). The sensitivity range of the ELISA was 15.6$1,000 \mathrm{ng} \cdot \mathrm{L}^{-1}$, and samples with concentrations above this range were reanalysed after dilution.

\section{Statistical evaluation}

In order to investigate the behaviour of various predictors of developing BOS, measurements were repeated over a period of 2 yrs. To adjust for repeated measures, regressions for each patient over time were fitted and coefficients in the BOS group $(n=5)$ were compared with those in the patients without BOS $(n=20)$ using the Wilcoxon rank test in order to avoid distribution problems of the regression estimators.

The effect of $\mathrm{OB}$, acute rejection or no rejection on variables in biopsy samples was taken into consideration by first averaging the variable levels from each biopsy episode and patient over the period. Biopsy results were then compared within patients using an analysis of variance model on ranked data, and mean differences were further analysed using Duncan's multiple range test [15]. The same test procedure was used to investigate predictor behaviour according to various types of infection. Correlations were assessed using the Spearman Rank test.

Results are expressed as medians and interquartile ranges (IQR, signifying the 25th and 75th percentiles) in tables and text, and as arithmetic mean \pm SEM in longitudinal graphs. Graphical comparison between groups with OB, acute rejection or no rejection are illustrated as box plots displaying the 10th, 25th, 50th, 75th and 90th percentiles of a variable. A p-value $<0.05$ was considered statistically significant.

\section{Results}

In the 25 patients, TBBs and BAL fluid samples were obtained from a total of 266 bronchoscopy procedures, of which 67 were performed because of clinical indications and 199 according to protocol.

\section{Acute rejection and $B O S$}

In $45(17 \%)$ TBB samples, no diagnosis of rejection could be made due to histological changes caused by reperfusion injury in the early postoperative period $(n=7)$ or nonspecific alveolitis $(\mathrm{n}=9)$, the scarcity of biopsy material $(n=7)$ or concomitant CMV pneumonitis $(n=22)$. Once these had been excluded, there were 98 histopathologically diagnosed acute rejection episodes of which 59 were classified as of minimal (A1), 35 mild (A2), and four moderate (A3) character. No episode of severe (A4) acute rejection was diagnosed.

Five patients developed BOS that was initially classified as 1 a ( $>20 \%$ reduction in FEV1 from baseline value [3]). After diagnosis of BOS was proven, all patients received augmented immunosuppressive treatment consisting of ATG therapy, additional methylprednisolone courses $(1 \mathrm{~g}$ i.v. for 3 days) and long-term treatment with high-dose inhaled budesonide $(800 \mu \mathrm{g}$ b.i.d.). In addition, two patients were converted from azathioprine therapy to methotrexate, and one from cyclosporine therapy to FK 506. In spite of these efforts, BOS in all five patients progressed over time and resulted in the histopathological changes of $\mathrm{OB}$ in $\mathrm{TBB}$ specimens. Two patients eventually became classified as BOS $2 \mathrm{~b}$ and three as BOS $3 \mathrm{~b}$.

The mean time from transplantation until diagnosis of BOS was 10 months (median 9, range 5-18 months). As a 
group, the patients with BOS had a higher number of acute rejections (mean 4.6) than those without BOS (mean 3.3), but this difference was not statistically significant. The incidence of CMV pneumonitis was comparable between the groups.

Of forty-three TBBs and BALs prospectively sampled in the BOS patients, $20(47 \%)$ were taken at intervals after BOS grade la to $3 \mathrm{~b}$ had been diagnosed (three episodes with concomitant CMV infection excluded). BAL differential cell counts in these 20 BOS samples showed significantly elevated percentages of neutrophils (mean 34.9, median 30.0, IQR 15.0-48.8) compared to acute rejection episodes (mean 11.9, median 4.5, IQR 2.0-12.6) as well as in patients with no rejection (mean 9.2, median 5.5, IQR 3.0$9.0),(\mathrm{p}<0.01$, table 2). There was a corresponding lower percentage of alveolar macrophages in patients with BOS compared to the other two groups, but no significant differences in percentages of eosinophils or lymphocytes (table 2).

The percentage of BAL volume return was significantly lower in the BOS samples (mean 42.5, median 48.0, IQR $43.0-58.5$ ) than in the acute rejection episodes (mean 58.6, median 61.0, IQR 50.8-63.8) as well as in patients with no rejection (mean 55.2, median 50.0, IQR 48.0-64.0), ( $\mathrm{p}<$ $0.05)$.

Bronchoalveolar lavage inflammatory markers in relation to acute rejection and bronchiolitis obliterans syndrome

The levels of MPO (mean $359 \mu \mathrm{g} \cdot \mathrm{L}^{-1}$, median 15, IQR 4-44), IL-8 (mean $184 \mathrm{ng} \cdot \mathrm{L}^{-1}$, median 52, IQR 5-50), ECP (mean $6.9 \mu \mathrm{g} \cdot \mathrm{L}^{-1}$, median 1.6, IQR $0-3.2$ ) and albumin (mean $88 \mathrm{mg} \cdot \mathrm{L}^{-1}$, median 61, IQR 41-103) were higher when acute rejection episodes were compared to no rejection (mean MPO $149 \mu \mathrm{g} \cdot \mathrm{L}^{-1}$, median 18, IQR 2-42); mean IL-8 $174 \mathrm{ng} \cdot \mathrm{L}^{-1}$, median 70, IQR 7-215; mean ECP $4.8 \mu \mathrm{g} \cdot \mathrm{L}^{-1}$, median 1.4 , IQR $0-1.8$; mean albumin 69 $\mathrm{mg} \cdot \mathrm{L}^{-1}$, median 51, IQR 33-76). However, none of these differences were statis-tically significant when repeated measures and infection had been accounted for (fig. 1) nor were any statistically significant differences in levels of inflammatory markers found between the different degrees of acute rejection (data not shown).

BOS patients had significantly higher levels of MPO (mean 2,683 $\mu \mathrm{g} \cdot \mathrm{L}^{-1}$, median 573, IQR 350-3,850, $\mathrm{p}<$ 0.001 ), IL-8 (mean 1,310 ng. $\mathrm{L}^{-1}$, median 751, IQR 751$1,750, \mathrm{p}<0.01$ ) and ECP (mean $60.4 \mu \mathrm{g} \cdot \mathrm{L}^{-1}$, median 43.7 , IQR 13.1-97.3, $\mathrm{p}<0.001$ ) compared to patients without BOS irrespective of their acute rejection status (figs. 1 and
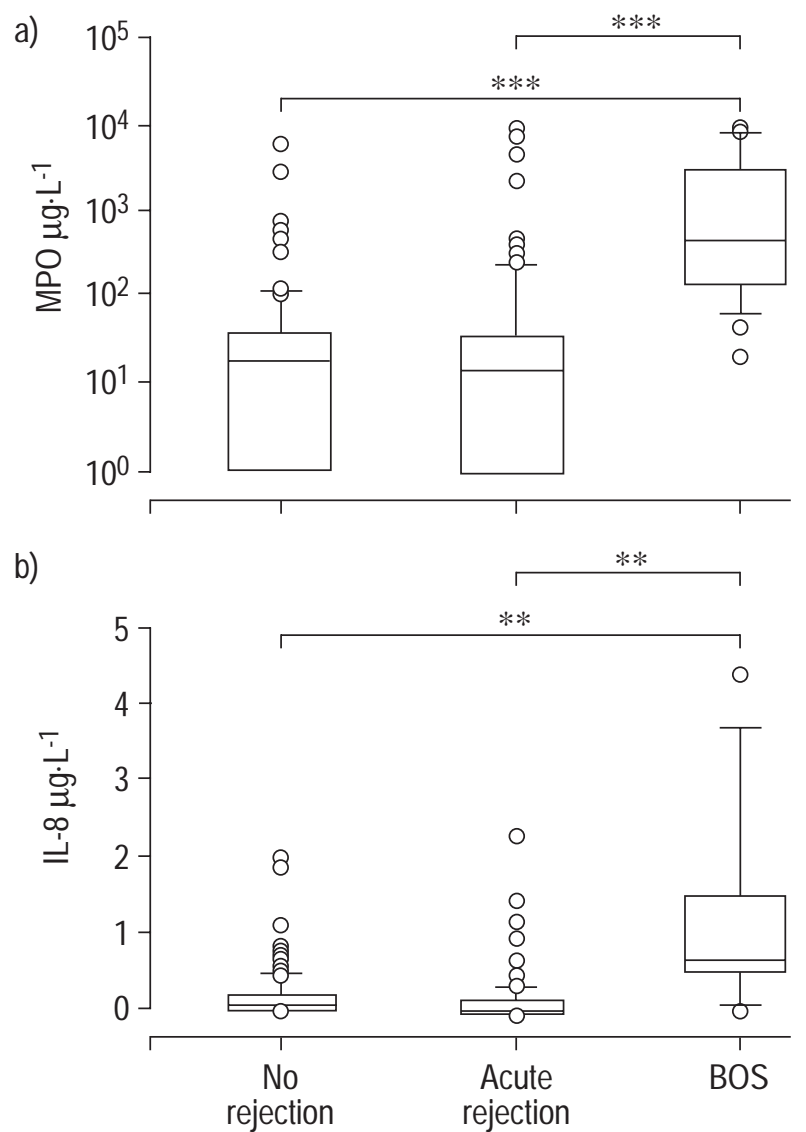

Fig. 1. - Bronchoalveolar lavage (BAL) fluid levels of: a) myeloperoxidase (MPO); and b) interleukin-8 (IL-8) in 20 BAL samples from five patients with bronchiolitis obliterans syndrome (BOS) grade 1 a to $3 \mathrm{~b}, 82$ BAL samples from all 25 patients with acute rejection grade A1 to A3 and 95 BAL samples from patients with no rejection (all infectious episodes excluded). Data are presented as box plots showing the median (50th percentile) and 25 th and 75 th percentiles, with the vertical bars representing the 10th and 90th percentiles. Outliers are also shown. $* *$ : $\mathrm{p}<0.01 ; * * *: \mathrm{p}<0.001$.

2). BOS levels of albumin (mean $59 \mathrm{mg} \cdot \mathrm{L}^{-1}$, median 44 , IQR 26-58) did not differ significantly from those of the other groups (fig. 2). Of the MPO, IL-8 and ECP samples in the outlying range above the 90 th percentile, $50 \%$ came from subjects who did not later develop BOS (eight of 16 samples, figs. 1 and 2).

When the levels of inflammatory markers were studied longitudinally (infection excluded), the five patients with BOS were found to have persistently elevated levels of MPO, ECP and to some extent IL-8 as compared to the 20

Table 2. - Median differential cell counts in bronchoalveolar lavage samples from patients with BOS grade $1 \mathrm{a}$ to $3 \mathrm{~b}$, acute rejection grade $A 1$ to $A 3$ and with no rejection

\begin{tabular}{|c|c|c|c|c|c|c|c|c|}
\hline & \multicolumn{8}{|c|}{ Differential cell count $\%$} \\
\hline & \multicolumn{2}{|c|}{ Neutrophils } & \multicolumn{2}{|c|}{ Eosinophils } & \multicolumn{2}{|c|}{ Lymphocytes } & \multicolumn{2}{|c|}{ Macrophages } \\
\hline & Median & IQR & Median & IQR & Median & IQR & Median & IQR \\
\hline $\mathrm{BOS}^{+}$ & $30.0^{* *}$ & $15.0-48.8$ & 0.0 & 0.0 & 6.3 & $2.5-12.5$ & 60.8 & $45.1-71.4$ \\
\hline Rejection $\#$ & 4.5 & $2.0-12.6$ & 0.0 & 0.0 & 12.0 & $4.2-20.0$ & 80.0 & $64.2-88.8$ \\
\hline No rejection ${ }^{\S}$ & 5.5 & $3.0-9.0$ & 0.0 & 0.0 & 7.0 & $4.1-13.1$ & 85.5 & $76.0-91.0$ \\
\hline
\end{tabular}

: all infectious episodes excluded; ${ }^{+}: 20$ samples, five patients; ${ }^{\#}: 82$ samples, 25 patients; ${ }^{\S}: 95$ samples. IQR: interquartile range. ${ }^{* *}$ : $\mathrm{p}<0.01$ versus patients without BOS. 


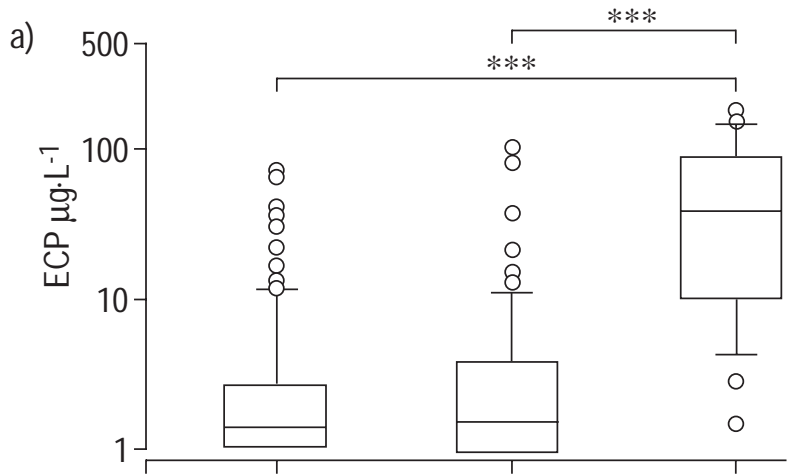

b)

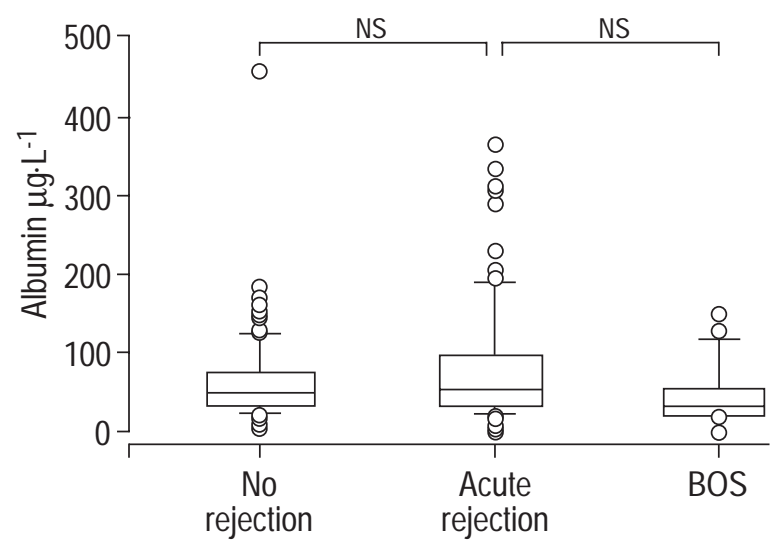

Fig. 2. - Bronchoalveolar lavage (BAL) fluid levels of: a) eosinophil cationic protein (ECP); and b) albumin in $20 \mathrm{BAL}$ samples from five patients with bronchiolitis obliterans syndrome (BOS) grade 1a to 3b, 82 BAL samples from all 25 patients with acute rejection grade A1 to A3 and $95 \mathrm{BAL}$ samples from patients with no rejection (all infectious episodes excluded). Data are presented as box plots showing the median (50th percentile) and 25th and 75th percentiles, with the vertical bars representing the 10th and 90 th percentiles. Outliers are also shown. ***: $\mathrm{p}<0.001$.

patients without BOS (figs. 3 and 4). This increase was significant for all three variables (MPO $\mathrm{p}<0.05, \mathrm{ECP}$ and IL-8 $\mathrm{p}<0.01)$. In four patients with BOS, the increase in MPO and ECP concentration preceded the clinical diagnosis of BOS by several months $(2,3,5$ and 5 months respectively). In the fifth patient, the increase occurred simultaneously with diagnosis of BOS (fig. 3). A successive lowering of FEV1 values (in per cent of best posttransplant value) over time was observed for the patients with BOS $(p<0.001$, fig. 3$)$. Mean percentages of BAL neutrophils were significantly elevated in BOS patients $(p<0.05)$ and followed a pattern close to that of IL-8 (data not shown).

\section{Infection}

Forty-three $(16 \%)$ samples were excluded from the analysis of infection due to histological changes caused by reperfusion injury in the early post-operative period $(n=7)$ or nonspecific alveolitis $(n=9)$, the scarcity of biopsy material $(n=7)$ or concomitant diagnosis of BOS $(n=23)$.

Nine episodes of bacterial infection, 19 of CMV pneumonitis, nine of PCP, two of Aspergillus fumigatus infection and two of RSV pneumonitis were diagnosed.

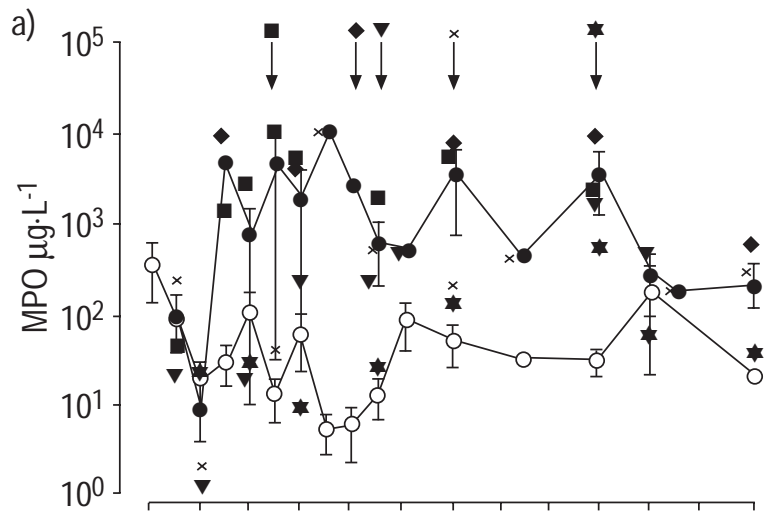

b)

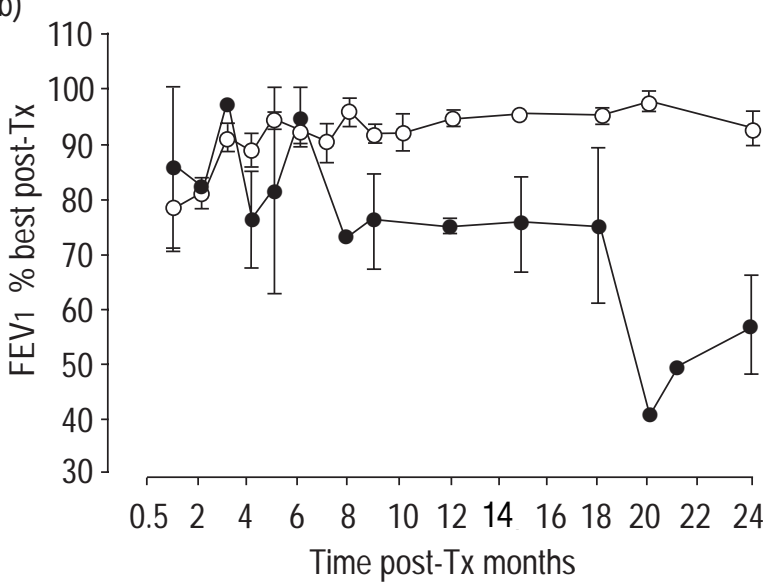

Fig. 3. - Longitudinal presentation of bronchoalveolar lavage fluid levels of: a) myeloperoxidase (MPO); and b) forced expiratory volume in one second (FEV1) in relation to time post-transplant (post-TX). Data are presented as mean \pm SEM and infectious episodes have been excluded. : bronchoscopic sampling episodes $(n=43)$ in five patients who developed bronchiolitis obliterans syndrome (BOS); $\bigcirc$ : sampling episodes $(n=154)$ in 20 patients without BOS. The different symbols represent individual MPO values in the five patients who developed BOS; the arrows indicate the tme of histopathological diagnosis of BOS for those patients.

All were treated adequately. With regard to low-grade bacterial colonization, one of the five patients who developed BOS had signs of this evidenced by low numbers of Pseudomonas aeruginosa in BAL cultures when the patient's BOS had deteriorated to stage $2 \mathrm{~b}$.

Bacterial infection resulted in significantly increased percentages of neutrophils (mean 25.3, median 23.5, IQR 8.5-32.0) compared to no infection (mean 10.4, median 5.0, IQR 2.2-9.6) $(\mathrm{p}<0.01)$ (table 3). Mean levels of ECP $\left(37.5 \mu \mathrm{g} \cdot \mathrm{L}^{-1}\right.$, median 13.7, IQR 7.2-69.0) and IL-8 $(1,679$ $\mathrm{ng} \cdot \mathrm{L}^{-1}$, median 770 , IQR $\left.607-2,950\right)$ were significantly elevated compared to no infection $(\mathrm{p}<0.01)$ (table 4$)$. This was not the case for MPO $\left(488 \mu \mathrm{g} \cdot \mathrm{L}^{-1}\right.$, median 319 , IQR 222-688) versus (244 $\mu \mathrm{g} \cdot \mathrm{L}^{-1}$, median 17 , IQR 0-38).

CMV pneumonitis was associated with increased percentages of neutrophils (mean 13.2, median 4.5, IQR 3.011.3) and elevated mean levels of inflammatory BAL markers, but none of these differences were statistically significant compared to no infection (tables 3 and 4).

PCP resulted in significantly increased percentage of BAL lymphocytes (mean 30.6, median 34.0, IQR 14.339.7) compared to no infection (mean 11.9, median 7.0, IQR 4.0-16.0) $(\mathrm{p}<0.01)($ table 3$)$ and elevated mean levels of 
Table 3. - Bronchoalveolar lavage fluid differential cell counts in relation to type of infection or the absence of infection*

\begin{tabular}{|c|c|c|c|c|c|c|c|c|c|}
\hline & \multirow[b]{3}{*}{ Subject $n$} & \multicolumn{8}{|c|}{ Differential cell count $\%$} \\
\hline & & \multicolumn{2}{|c|}{ Neutrophils } & \multicolumn{2}{|c|}{ Eosinophils } & \multicolumn{2}{|c|}{ Lymphocytes } & \multicolumn{2}{|c|}{ Macrophages } \\
\hline & & Median & IQR & Median & IQR & Median & IQR & Median & IQR \\
\hline No infection & 179 & 5.0 & $2.2-9.6$ & 0.0 & 0.0 & 7.0 & $4.0-16.0$ & 82.0 & $71.0-90.0$ \\
\hline Bacteria & 9 & $23.5^{* *}$ & $8.5-32.0$ & 0.0 & 0.0 & 6.0 & $3.9-9.2$ & 67.5 & $48.1-79.5$ \\
\hline CMV pneumonitis & 19 & 4.5 & $3.0-11.3$ & 0.0 & 0.0 & 10.5 & $4.5-15.5$ & 80.5 & $68.5-85.0$ \\
\hline PCP & 9 & 6.0 & $3.2-20.0$ & 0.0 & $0.0-1.0$ & $34.0 * *$ & $14.7-39.7$ & 56.0 & $42.7-70.0$ \\
\hline Aspergillus & 2 & 29.8 & $6.9-54.4$ & 0.0 & 0.0 & 7.5 & $2.5-10.0$ & 62.8 & $42.0-84.5$ \\
\hline RSV pneumonitis & 2 & 31.0 & $1.0-61.0$ & 0.3 & $0.0-0.5$ & 9.3 & $8.5-10.0$ & 59.5 & $29.0-90.0$ \\
\hline
\end{tabular}

*: transbronchial lung biopsy samples indicating reperfusion injury $(n=7)$ or nonspecific alveolitis $(n=9)$, the scarcity of biopsy material $(\mathrm{n}=7)$ or concomitant diagnosis of bronchiolitis obliterans syndrome $(\mathrm{n}=23)$ were excluded. IQR: interquartile range; CMV: cytomegalovirus; PCP: Pneumocystis carinii pneumonia; Aspergillus: Aspergillus fumigatus infection; RSV: respiratory syncytial virus. ${ }^{* *}: \mathrm{p}<0.01$ versus no infection.

albumin (323 mg. $\mathrm{L}^{-1}$, median 114, IQR 90-223) compared to no infection (mean $77 \mathrm{mg} \cdot \mathrm{L}^{-1}$, median 56 , IQR 49-90) $(\mathrm{p}<0.01)($ table 4).

The incidence of Aspergillus $(\mathrm{n}=2)$ and RSV $(\mathrm{n}=2)$ infection was too low to draw any safe statistical conclusions, but RSV especially resulted in very high levels of all inflammatory markers including albumin (table 4).

\section{Correlations}

BAL percentage of neutrophils correlated with BAL levels of MPO $(\rho=0.58)$ and ECP $(\rho=0.59)$ and more weakly with levels of IL-8 $(\rho=0.42)$, (infection excluded).

FEV1 ( $\%$ of the predicted value) correlated weakly and negatively with BAL levels of MPO $(\rho=-0.34)$, ECP $(\rho=-$ $0.36)$ and IL-8 ( $\rho=-0.31)$ (infection excluded).

\section{Discussion}

This prospective longitudinal study in lung transplant recipients shows that attraction and activation of the neutrophil granulocyte, assessed by levels of IL-8, MPO and ECP in BAL fluid, is associated with development of BOS. In four of five patients with BOS, a continuous elevation of MPO and ECP levels as well as percentage of neutrophils in BAL fluid was observed to precede the clinical diagnosis of BOS by several months.

The pathophysiological process behind OB in BOS involves a number of cell types, leading to subepithelial scarring of the respiratory bronchioles with fibrotic granulation tissue eventually occluding the lumen [3]. In analogy with acute rejection, research has focused on the T-lymphocyte in connection with BOS, but no major differences in T-cell subpopulations have been found [16]. In contrast, there is mounting evidence that the neutrophil granulocyte is an important cell associated with BOS. Increased percentages of BAL fluid neutrophils have been reported [16, 17], and increased numbers of BAL fluid neutrophils as well as BAL fluid levels of IL-8 seem to be a feature of BOS. DiGiovine et al. [10] performed chemotactic studies in which IL-8 appeared to account for the neutrophil presence, and IL- 8 was also found in the peribronchial region of their transbronchial biopsy specimens. Furthermore, in a cross-sectional study evidence was recently found of neutrophil granulocyte activation in the form of increased BAL fluid levels and degree of immunohistochemical staining of MPO in TBBs from BOS patients compared to matching controls [9].

In the present study, the longitudinal pattern of granulocyte activation markers was investigated in connection with development of BOS. MPO, an oxidative enzyme released from primary neutrophil granules, has been found previously to be increased in conditions characterized by neutrophil airway inflammation such as chronic bronchitis and chronic obstructive pulmonary disease (COPD) [18, 19]. Furthermore, in BOS patients, BAL fluid MPO levels were approximately 20 times higher than those reported in COPD patients [20], and remained high during the observation period. MPO is involved in the production

Table 4. - Bronchoalveolar lavage fluid inflammatory markers in relation to type of infection or the absence of infection*

\begin{tabular}{|c|c|c|c|c|c|c|c|c|c|}
\hline & \multirow{3}{*}{$\begin{array}{c}\text { Subject } \\
n\end{array}$} & \multicolumn{8}{|c|}{ Inflammatory marker } \\
\hline & & \multicolumn{2}{|c|}{ MPO $\mu \mathrm{g} \cdot \mathrm{L}^{-1}$} & \multicolumn{2}{|c|}{ ECP $\mu \mathrm{g} \cdot \mathrm{L}^{-1}$} & \multicolumn{2}{|c|}{ IL-8 $\mu \mathrm{g} \cdot \mathrm{L}^{-1}$} & \multicolumn{2}{|c|}{ Albumin $\mathrm{mg} \cdot \mathrm{L}^{-1}$} \\
\hline & & Median & IQR & Median & IQR & Median & IQR & Median & IQR \\
\hline No infection & 179 & 17 & $0-38$ & 1.5 & $0.0-3.4$ & 60 & 20-195 & 56 & $49-90$ \\
\hline Bacteria & 9 & 319 & $222-688$ & $13.7 * *$ & $7.2-69.0$ & $770 * *$ & $607-2950$ & 56 & $41-85$ \\
\hline CMV pneumonitis & 19 & 13 & $0-215$ & 0.2 & $0.0-7.2$ & 109 & $18-282$ & 56 & 39-102 \\
\hline PCP & 9 & 24 & $0-30$ & 2.1 & $1.4-2.8$ & 140 & $65-205$ & $114 * *$ & $90-223$ \\
\hline Aspergillus & 2 & 415 & 20-792 & 13.9 & $0.0-26.1$ & 6456 & $212-500$ & 54 & $31-80$ \\
\hline RSV pneumonitis & 2 & 2186 & $1420-3050$ & 114.2 & $103-141$ & 11260 & $1320-20000$ & 636 & $182-1130$ \\
\hline
\end{tabular}

*: transbronchial lung biopsy samples indicating reperfusion injury $(\mathrm{n}=7)$ or nonspecific alveolitis $(\mathrm{n}=9)$, the scarcity of biopsy material $(n=7)$ or concomitant diagnosis of bronchiolitis obliterans syndrome $(n=23)$ were excluded. MPO: myeloperoxidase; ECP: eosinophil cationic protein; IL-8: interleukin-8; IQR: interquartile range; CMV: cytomegalovirus; PCP: Pneumocystis carinii pneumonia; Aspergillus: Aspergillus fumigatus infection; RSV: respiratory syncytial virus. ${ }^{* *}$ : $\mathrm{p}<0.01$ versus no infection. 

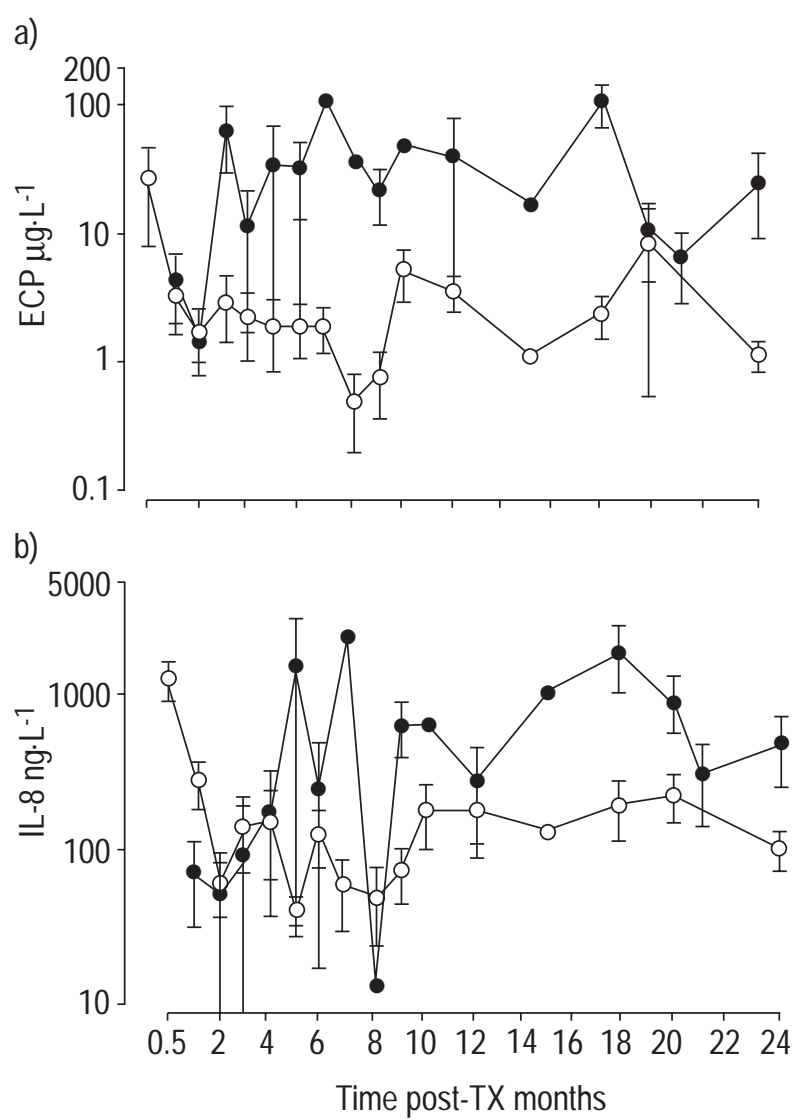

Fig. 4. - Longitudinal presentation of bronchoalveolar lavage fluid levels of: a) eosinophil cationic protein (ECP); and b) interleukin-8 (IL8 ) in relation to time post-transplant (post-TX). Data are presented as mean \pm SEM and infectious episodes have been excluded. $\bullet$ : bronchoscopic sampling episodes $(n=43)$ in five patients who developed bronchiolitis obliterans syndrome (BOS); $\bigcirc$ : sampling episodes $(n=154)$ in 20 patients without BOS.

of hypochlorous acid, a powerful oxidant, which together with neutrophil-derived proteases can increase oxidative stress in the airways [21]. This is likely to be detrimental to allograft function, and indications from an earlier study pointed towards increased consumption of antioxidative defences in BOS patients, which correlated with high BAL fluid neutrophil percentages [9].

ECP, a cytotoxic eosinophil granule protein usually associated with the eosinophilic inflammation found in asthma [22] is also produced by neutrophil granulocytes at $\sim 1 \%$ of eosinophil production [23]. Since the present BAL fluid ratio of ECP to MPO was of a magnitude of 1:100, it is probable that the observations of increased levels of MPO and ECP in BOS are both expressions of the same phenomenon, namely neutrophil granulocyte-activation. The similarities in the longitudinal pattern of MPO and $\mathrm{ECP}$, the evident increase in BAL fluid neutrophil percentage compared to the lack of changes in eosinophil percentage, and the strong BAL fluid neutrophil correlation with both MPO and ECP levels are all indications supporting this view.

In accordance with earlier findings, the process of BOS appears to involve IL-8, a cytokine with a known chemotactic effect on neutrophil granulocytes [24] and produced mainly by alveolar macrophages and the respiratory epithelium [25]. IL-8 induces neutrophil degranulation and production of oxygen radicals, and although these aspects were not investigated in the present study, the increased levels of IL- 8 in BOS suggest that oxidative stress may play a role. Levels of IL-8 were significantly increased in the BOS patients from the 5th post-operative month and continued at a high level during the rest of the observation period. In analogy with other chronic inflammatory disorders, it is possible that IL-8 constitutes a long-term signal responsible for neutrophil influx and activation in the airways of lung transplant recipients with OB. Other chemotaxins such as leukotriene $\mathrm{B}_{4}$ [26] may also be involved in this process, but this was not assessed in the present study.

No significant impact of acute rejection on the investigated variables was found, irrespective of degree of acute rejection, a finding in accordance with earlier results [8]. This indicates that granulocytes, in contrast to T-lymphocytes, do not seem to play an important role in the process of acute lung rejection. However, it should be pointed out that the majority of the acute rejections in the study were of minimal or mild character, and this could have affected the results.

A possible confounding factor in the study is the influence of concomitant infection on inflammatory cells and markers, and infectious episodes were therefore analysed separately from acute and chronic rejection situations. Any subclinical bacterial infection in the patients was excluded due to a thorough clinical and bacteriological monitoring. As might be expected, bacterial infection went together with significant neutrophilia and increased mean levels of both ECP and IL-8. Episodes of manifest CMV pneumonitis, however, showed only moderately elevated levels of inflammatory cells and markers not statistically different from episodes with no infection. To the authors' knowledge, inflammatory biomarker reactions in CMV pneumonitis have not been studied in great detail, although CRAIGEN et al. [27] recently reported increased IL-8 production and enhanced neutrophil transendothelial migration in a fibroblast in vitro model. A clinical study in renal transplant recipients found no increase in urinary levels of MPO or CRP during CMV infection of the allograft [28]. It is therefore too early to draw any conclusions from the analysis of soluble biomarkers about the character of the inflammatory process in $\mathrm{CMV}$ infection.

No increased incidence of CMV pneumonitis was found in the BOS group, which could be due to the small size of the study group. Others have found CMV to be clearly associated with an increased risk of developing BOS [4]. Episodes of PCP were associated with a significantly increased BAL fluid percentage of lymphocytes as well as levels of albumin indicating ongoing vascular leakage of plasma. However, neither BAL neutrophilia nor increase in IL-8 concentration as reported in PCP in acquired immune deficiency syndrome patients was found in the present study [29].

Neither the mean age nor the ratio of single lung, bilateral lung and heart/lung transplant operations differed significantly between the BOS patients and the transplant recipients without BOS. The sex ratio was oblique with the BOS group having only female patients, but female sex is not known to be a factor of importance for development of BOS. Augmented immunosuppressive therapy was attempted in all BOS patients, but had no apparent impact on longitudinal levels of analysed variables nor on the disease 
progress. There has been one report that the enzymatic activity of MPO is impaired by the immunosuppressant azathioprine, but no data is available regarding any influence on the levels of the enzyme [30]. The two patients in the present study who were converted from azathioprine to methotrexate after BOS had developed did not show any change in MPO levels associated with the therapy change.

The present results indicate that, in the absence of bacterial infection, measurements of the granulocyte activation markers myeloperoxidase and eosinophil cationic protein in bronchoalveolar lavage fluid could have potential as early predictors of chronic rejection and obliterative bronchiolitis in lung transplant recipients. The finding of continually elevated myeloperoxidase levels and percentages of neutrophils in bronchoalveolar lavage fluid over time lend support to the diagnosis of bronchiolitis obliterans syndrome.

Acknowledgements. The authors thank B Areskoug for statistical consultations.

\section{References}

1. Hosenpud JD, Novick RJ, Bennett LE, Keck BM, Fiol B, Daily OP. The Registry of the International Society for Heart and Lung Transplantation: thirteenth official report - 1996. J Heart Lung Transplant 1996; 15: 655-674.

2. Bando K, Paradis I, Similo S, et al. Obliterative bronchiolitis after lung and heart-lung transplantation. $J$ Thoracic Cardiovasc Surg 1995; 110: 4-14.

3. Cooper JD, Billingham M, Egan $\mathrm{T}$, et al. A working formulation for the standardization of nomenclature and for clinical staging of chronic dysfunction in lung allografts. J Heart Lung Transplant 1993; 12: 713-716.

4. Sharples LD, Tamm M, McNeil K, Higenbottam TW, Stewart S, Wallwork J. Development of bronchiolitis obliterans syndrome in recipients of heart/lung transplantation - early risk factors. Transplantation 1996; 61: 560-566.

5. Kshettry VR, Kroshus TJ, Savik K, Hertz MI, Bolman RM. Primary pulmonary hypertension as a risk factor for the development of obliterative bronchiolitis in lung allograft recipients. Chest 1996; 110: 704-709.

6. Kramer MR, Stoehr C, Whang JL, et al. The diagnosis of obliterative bronchiolitis after heart/lung and lung transplantation: low yield of transbronchial lung biopsy. J Heart Lung Transplant 1993; 12: 675-681.

7. Reichenspurner $\mathrm{H}$, Girgis RE, Robbins RC, et al. Stanford experience with obliterative bronchiolitis after lung and heart/lung transplantation. Ann Thorac Surg 1996; 62: 1467-1472. Discussion 1472-1473.

8. Riise GC, Kjellström C, Ryd W, et al. Inflammatory cells and activation markers in BAL during acute rejection and infection in lung transplant recipients: a prospective, longitudinal study. Eur Respir J 1997; 10: 1742-1746.

9. Riise GC, Williams A, Kjellström C, Scherstén H, Andersson BA, Kelly F. Bronchiolitis obliterans syndrome in lung transplant recipients is associated with increased neutrophil activity and decreased antioxidant status in the lung. Eur Respir J 1998; 12: 82-88.

10. DiGiovine B, Lynch JPr, Martinez FJ, et al. Bronchoalveolar lavage neutrophilia is associated with obliterative bronchiolitis after lung transplantation: role of IL-8. J Immunol 1996; 157: 4194-4202.

11. Riise GC, Scherstén H, Nilsson FN, Ryd W, Andersson B. Activation of eosinophils and fibroblasts assessed by eosinophil cationic protein and Hyaluronan in BAL. Association with acute rejection in lung transplant recipients. Chest 1996; 110: 89-96.
12. Scherstén H, Hedner T, McGregor CGA, et al. Increased levels of endothelin-1 in bronchoalveolar lavage fluid of patients with lung allografts. $J$ Thorac Cardiovasc Surg 1996; 111: 253-258.

13. Yousem SA, Berry G, Brunt E, et al. A working formulation for the standardization of nomenclature in the diagnosis of heart and lung rejection: lung rejection study group. J Heart Transplant 1990; 9: 593-601.

14. Yousem S, Berry G, Caigle P, et al. Revision of the 1990 working formulation for the classification of pulmonary allograft rejection: lung rejection study group. $J$ Heart Lung Transplant 1996; 15: 1-15.

15. Duncan D. t-tests and intervals for comparisons suggested by the data. Biometrics 1975; 31: 339-359.

16. Maurer JR, Gouch E, Chamberlain DW, Patterson GA, Grossman RF. Sequential bronchoalveolar lavage studies from patients undergoing double lung and heart/lung transplant. Transplant Proc 1989; 21: 2585-2587.

17. Griffith BP, Paradis IL, Zeevi A, et al. Immunologically mediated disease of the airways after pulmonary transplantation. Ann Surg 1988; 208: 371.

18. Riise GC, Ahlstedt S, Larsson S, et al. Bronchial inflammation in chronic bronchitis assessed by measurement of cell products in bronchial lavage fluid. Thorax 1995; 50: 360-365.

19. Keatings VM, Barnes PJ. Granulocyte activation markers in induced sputum: comparison between chronic obstructive pulmonary disease, asthma, and normal subjects. $\mathrm{Am}$ J Respir Crit Care Med 1997; 155: 449-453.

20. Linden M, Rasmussen JB, Piitulainen E, et al. Airway inflammation in smokers with nonobstructive and obstuctive chronic bronchitis. Am Rev Respir Dis 1993; 148: 1226-1232.

21. Weiss S. Tissue destruction by neutrophils. New Engl $J$ Med 1989; 320: 365-376.

22. Bousquet J, Chanez P, Lacoste J, et al. Eosinophilic inflammation in asthma. N Engl J Med 1990; 323: 10331039.

23. Abu-Ghazaleh RI, Dunnette SL, Loegering DA, et al. Eosinophil granule proteins in peripheral blood granulocytes. J Leukoc Biol 1992; 52: 611-618.

24. Richman-Eisenstat JBY, Jorens PG, Hebert CA, Ueki I, Nadel JA. Interleukin-8: an important chemoattractant in sputum of patients with chronic inflammatory airway diseases. Am J Physiol 1993; 264: L413-L418.

25. Baggiolini M, Walz A, Kunkel SL. Neutrophil-activating peptide-1/interleukin-8, a novel cytokine that activates neutrophils. J Clin Invest 1989; 84: 1045-1049.

26. Martin T, Altman L, Albert R, Henderson W. Leukotriene B4 production by the human alveolar macrophage: a potential mechanism for amplifying inflammation in the lung. Am Rev Respir Dis 1984; 129: 106-111.

27. Craigen JL, Yong KL, Jordan NJ, et al. Human cytomegalovirus infection up-regulates interleukin-8 gene expression and stimulates neutrophil transendothelial migration. Immunology 1997; 92: 138-145.

28. Steinhoff J, Einecke G, Niederstadt C, et al. Renal graft rejection or urinary tract infection? The value of myeloperoxidase, C-reactive protein, and alpha2-macroglobulin in the urine. Transplantation 1997; 64: 443-447.

29. Benfield TL, Vestbo J, Junge J, Nielsen TL, Jensen AB, Lundgren JD. Prognostic value of interleukin-8 in AIDSassociated Pneumocystis carinii pneumonia. Am J Respir Crit Care Med 1995; 151: 1058-1062.

30. Murthy S, Anania T, Clearfield H. Azathioprine reduces extravasation and neutrophil trafficking in immune complex-mediated inflammation in the rat colon. Agents Actions 1991; 34: 244-250. 